\title{
Correction: The outcomes and prognostic factors of acute respiratory failure in the patients 90 years old and over
}

\section{Wan-Ling Chen ${ }^{1, *}$, Chin-Ming Chen ${ }^{2,3, *}$, Shu-Chen Kung ${ }^{1}$, Ching-Min Wang ${ }^{4}$, Chih- Cheng Lai ${ }^{5}$ and Chien-Ming Chao ${ }^{5,6}$}

${ }^{1}$ Department of Respiratory Therapy, Chi Mei Medical Center, Liouying, Tainan, Taiwan

2 Department of Intensive Care Medicine, Chi Mei Medical Center, Liouying, Tainan, Taiwan

3 Department of Recreation and Health-Care Management, Chia Nan University of Pharmacy \& Science, Tainan, Taiwan

${ }^{4}$ Department of Internal Medicine, Chi Mei Medical Center, Liouying, Tainan, Taiwan

${ }^{5}$ Department of Intensive Care Medicine, Chi Mei Medical Center, Liouying, Tainan, Taiwan

${ }^{6}$ Department of Nursing, Min-Hwei College of Health Care Management, Tainan, Taiwan

* These authors have contributed equally to this work

Published: March 20, 2018

Copyright: Chen et al. This is an open-access article distributed under the terms of the Creative Commons Attribution License 3.0 (CC BY 3.0), which permits unrestricted use, distribution, and reproduction in any medium, provided the original author and source are credited.

This article has been corrected: The proper name of the institutions are as follows:

${ }^{1}$ Department of Respiratory Therapy, Chi Mei Medical Center, Liouying, Tainan, Taiwan

2 Department of Intensive Care Medicine, Chi Mei Medical Center, Liouying, Tainan, Taiwan

${ }^{3}$ Department of Recreation and Health-Care Management, Chia Nan University of Pharmacy \& Science, Tainan, Taiwan

${ }^{4}$ Department of Internal Medicine, Chi Mei Medical Center, Liouying, Tainan, Taiwan

${ }^{5}$ Department of Intensive Care Medicine, Chi Mei Medical Center, Liouying, Tainan, Taiwan

${ }^{6}$ Department of Nursing, Min-Hwei College of Health Care Management, Tainan, Taiwan

* These authors have contributed equally to this work

Original article: Oncotarget. 2018; 9:7197-7203. https://doi.org/10.18632/oncotarget.24051 